\title{
Survey and comparison of different classification techniques for select appropriate classifier of image
}

\author{
Sahar Muneam ${ }^{1}$, Mohammad Q. Jawad ${ }^{2}$, Dina Hassan ${ }^{3}$ \\ ${ }^{1}$ Department of Computer Science, College of Science for women, University of Baghdad, Iraq \\ ${ }^{2}$ University of Information Technology and Communications \\ ${ }^{3}$ Department of Computer Science, College of Science for women, University of Baghdad, Iraq
}

\begin{tabular}{|c|c|}
\hline Article Info & ABSTRACT \\
\hline Received, 2019 & $\begin{array}{l}\text { In human visual system, visual object classification is easy and effortless but } \\
\text { in computer vision systems it is extremely hard Because of the various images } \\
\text { of different objects within a specific class may have together with the various } \\
\text { viewing conditions had led to have a serious problem. If some images have }\end{array}$ \\
\hline Keyword: & types of images. Images processing introduces several techniques which be \\
\hline Image classification & able to classify the data, but if image is blurry or noisy so they can not able to \\
\hline Feature extraction & give the acceptable results. In this survey discuss the main classification \\
\hline ANN & motivation of this survey is to gives a brief comparison among different \\
\hline SVM & images classification techniques and methods. Finally, it is determined \\
\hline DT & method that more accurately if an image contains blurry or noisy data. \\
\hline
\end{tabular}

\section{Corresponding Author:}

Mohammad Q. Jawad

University of Information Technology and Communications

Email : mohammad.qassim2002@uoitc.edu.iq

\section{Introduction}

The world is developing rapidly every day .All scientific fields have been affected by the rapid development of computer technology. The increase of high-powered computers, the availability of low-cost and high quality cameras, led to production of millions of images every day[1], each of these image requires their classification in way have generated an importance in image classification because represents a significant issue in many applications that involve detecting face, recognizing an optical character, or a finger print, classifying the abnormalities in medical images, and in any other surveillance purposes, industrial visual inspection, vehicle navigation, robot control, remote sensing, and many computer vision applications. Though, the manual process of classifying images is characterized by being time-consuming, and repetitive, and not always reliable. Therefore, the automatic process of image classification still represents an important need [2].

Image classification is a critical and it is extremely hard to recognize an object in an image. Especially when image contains, noise, Background clutter or bad feature. In this paper discuss major techniques of image classification and some other related technique. First technique supervised learning method can be used to label the data. In this method, every data point is of two parts: ('input' and 'output' or 'label'). The former includes the feature variables whereas the latter represents the desired value for this data point. 
Following this way of data organization, training algorithms can be run over the given input/output pairs. This helps build a model that will predict the labels of any future unlabeled test data [3]. Second technique Unsupervised learning when they obtained training data have no labels, the unsupervised learning method needs to be used. This will facilitate the process of inferences from the data in question. This method is statistics-based, but it can also be utilized in data mining. It helps searching for the patterns in the given data to be later grouped into different clusters [4]

There are many classification techniques that have been developed in order to perform the image classification process like the Decision Tree (DT), Artificial Neural Network (ANN), Support Vector Machine (SVM) and Fuzzy Logic.

\section{Related work}

Jianxin $\mathrm{Wu}$ et al. [1], the image classification challenges from a big dataset is the issue of the current research work. The support vector machine (SVM) classifier show to be very efficient in the image classification.

Fuliang Wang And Feng Wang et al. [5], explains Artificial Neural Network is efficiently managing the noisy data. Similarly, Monica Bianchini et al. [6] also discuss the artificial neural network classification technique. Shanmugam et al. [7], classified a natural war scene using ANN and SVM. Then, the results obtained from both methods were compared, and the best classified scene was determined, the rate of SVM classification was 59\% whereas that of ANN was $72.5 \%$. This result proves that ANN is better than SVM.

Ponce et al. [8], classifier is completed with SVM. This technique achieves high accurateness on a big database content natural scenes category that well known Caltech-101 dataset. The rate of classification is $72.2 \%$. All image classification methods have advantages and some disadvantages. Some methods are the combination of another classifier in image classification. A classifier is considered efficient if it has ability to correctly predict. Figure (1) shows the main steps of designing the intelligent classification system.

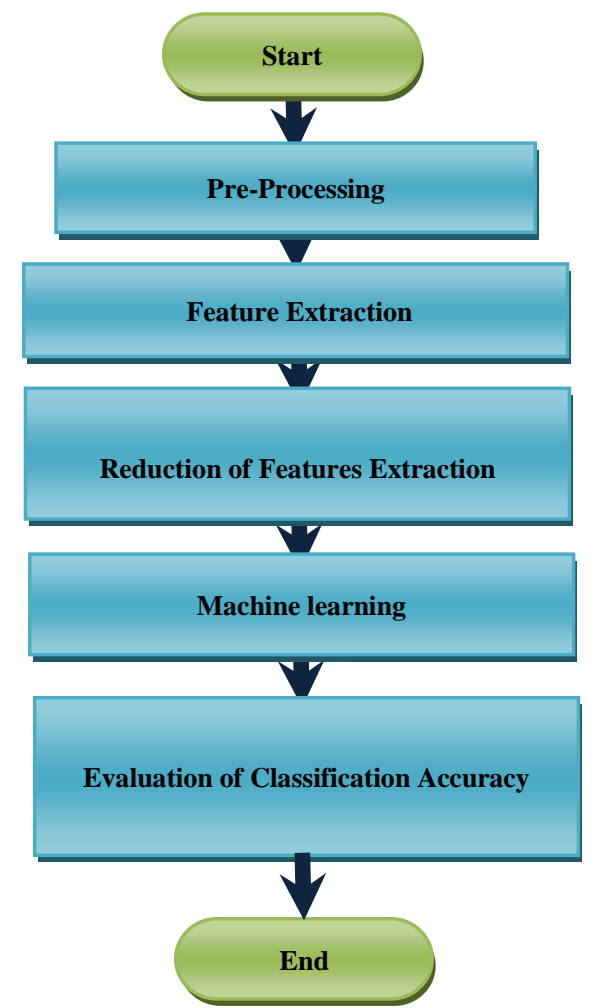

Figure 1. Main steps of designing the intelligent classification system. 


\section{General Steps of Image Classification}

Figure (1) illustrates a typical image classification system, which consists of the following blocks:

A. Pre-processing: This step involves processing the input data in advance before being fed into the feature extraction step. Pre-processing techniques include the following sub-processes: filtering, normalization, trimming, transformation, alignment, offset correcting, windowing, smoothing etc. However, the choice among these sub-processes depends on applications like: the intensity of the used brightness and color normality in face-recognition. Further, it may depend on the end-point detection for recognition of speech. Generally, these two techniques represent the most common ones.

B. Features Extraction: In this step, The process of extracting features can be implemented using one or more standard methods (mixing between two or more methods) like: Principle Component Analysis , Histograms of Oriented Gradients, Local Binary Pattern etc. Some of these features are significant for specific application according to the mathematical computation for each type of feature.

$C$. Reduction of Features Extraction: In general, the resulted features that are from the most standard methods of feature extraction are huge. Thus, specific technique must be applied to minimize the computed features to select strongest set of features to describe input image. Originally, this minimization issue is applied to produce limited number of features called active features as an input data to the intelligent classification system not the original input data (image). Also, increasing the number of working features in intelligent system may lead to more complexity to design this system and training stage for it has more time.

$D$. Machine learning: The images are classified based on the extracted features into predefined categories by using suitable methods.

E. Evaluation of Classification Accuracy: This step implies determining the performance of the system by evaluating the classification process.

\section{Image Features Extraction}

For object detection or classification, the image features need to hold a lot of information about the object of the image in question. Besides, it should further discard any irrelevant or redundant information about the extraction process. The computation needs to be easy so that the approach will be practical when there is a large collection of images, and rapid when conducting the extraction process. These stages reflect the perceptual characteristics of humans. Before the image is entered into the classification system, the image is captured by the camera. When the image captures, some information in the real world will automatically be lost. This information loss will due to several kinds of variations in image that have an effect on the classification, such as:

- Illumination - The change in lighting causes significant changes in the pixel intensity value. This factor affects the appearance of the objects in the images, making it difficult to classify or recognition object in the image Figure (2) illustrates this situation.

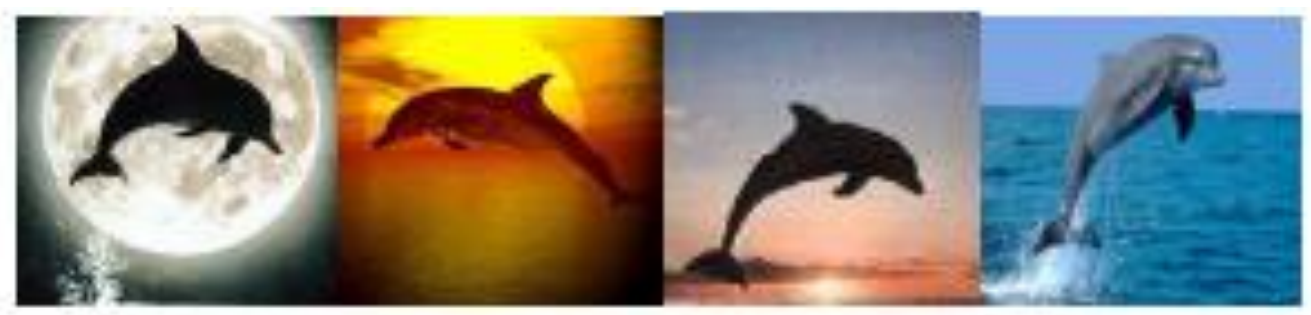

Figure (2): Illumination variation

- Size and Scale - In the images, the object can be appearance either in front or far. These effects can show the object is similar to other objects in new categories. Figure (3) illustrates this situation. 


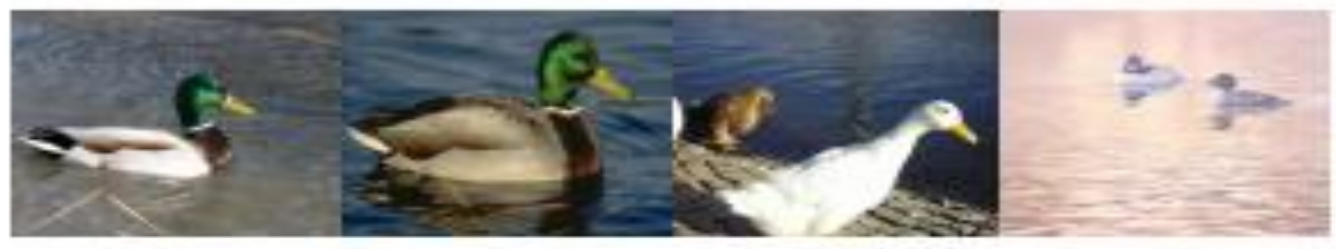

Figure(3): Size scale variation

- Background Clutter - In some images consists of a complex background. This generate confuses the confusion between the objects in the foreground and background image This problem increases the errors resulting in the process of image classification as shown in Figure(4).

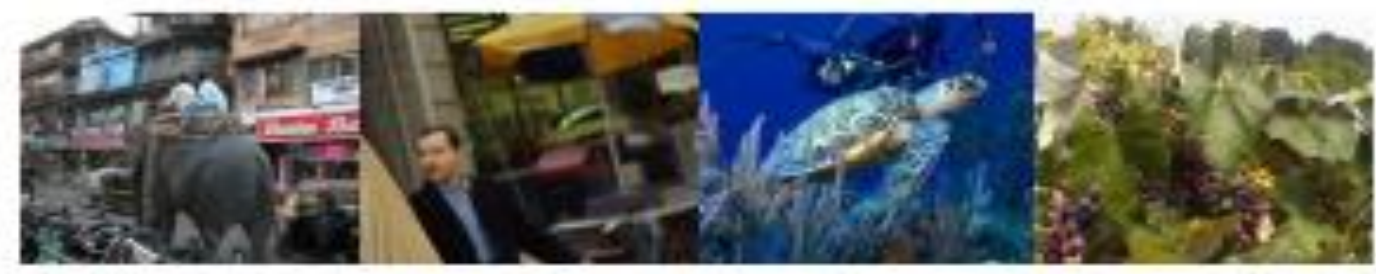

Figure (4): Background Clutter

- Viewpoint - The place of the camera can affect the object appearance of the image and this leads to different results in object classification as shown in Figure(5).

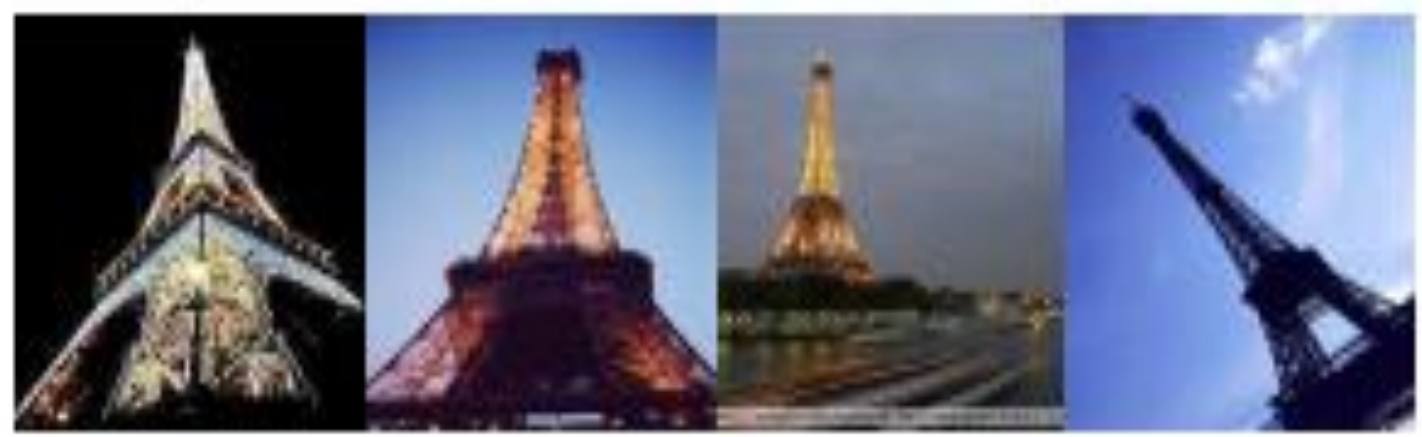

Figure (5): Viewpoint

- Intra-Class Variability - The difference between instances of objects belonging to the similar class.

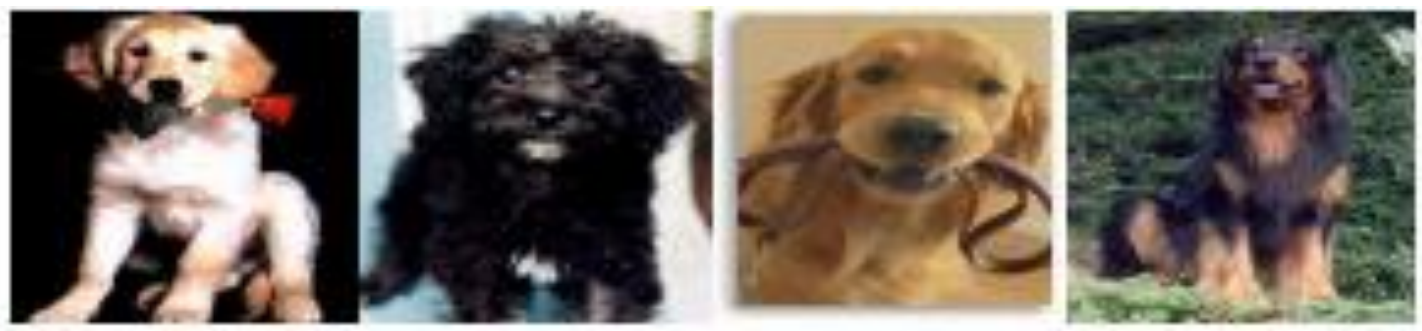

Figure (6): Intra-Class Variability.

This difference among real scene and image information is known sensory gap Figure ( 7) [9, 10]. In classification procedure the main lack is the difference among the visual features of image and the objects, meanings that this image as perceived by a human. This is named semantic gap and it is define as the lack of coincidence among the information that can extract from the visual data and the analysis that the same data from a user in given situation [9]. 


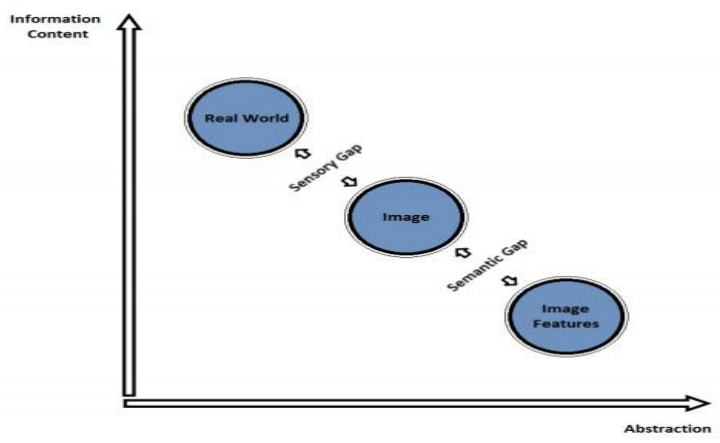

Figure 7. Sensor and semantic gap

In order to overcome these problems and reduce these gaps to obtain a high classification accuracy, one is required to use advance image feature descriptors and discard the direct application of raw image intensities or gradients. Such features depend on points, blobs, intensities, gradients, colour or their combinations. In a word, the final feature descriptor needs to represent the image sufficiently well for the detection and classification process.

\section{There are various kinds of feature extraction approaches:}

A. Color Features: Colors are an important feature in image classification [11,12]. The color histogram Method represent the most general way for extracting colored features It is consider as the distribution of color in image. The effectiveness of color feature that is independent and not sensitive to size, zoom and rotation of the image $[13,14]$.

B. Texture Features: The extraction of texture feature is very powerful technique, especially for a big image that have a repetitive area. The texture is a collection of pixel that have certain properties. This method can be classified into two classes: spectral texture feature extraction and spatial texture feature extraction $[14,15,16]$.

C. Shape Features: Shape features are used most often in ( shape description and object recognition). This method can be classified into: contour based and region based [16, 17]. The contour methods compute the features from boundary and do not take into account interior, whereas the region methods compute the features from the entrie region.

Table 1. Brief review to the famous descriptors that used in image classification

\begin{tabular}{|c|c|l|l|}
\hline $\begin{array}{c}\text { Descriptor } \\
\text { type }\end{array}$ & $\begin{array}{c}\text { Descriptor } \\
\text { name }\end{array}$ & \multicolumn{1}{|c|}{ Strength } & \multicolumn{1}{|c|}{ Weakness } \\
\hline Color & $\begin{array}{c}\text { Color } \\
\text { histogram }\end{array}$ & $\begin{array}{l}\text { It is uncomplicated to calculate, be able } \\
\text { to use for both global and local } \\
\text { descriptions of color, and is strong to } \\
\text { rotation, translation, viewing angle. }\end{array}$ & $\begin{array}{l}\text { The colors histograms do not capture } \\
\text { the spatial information of pixel, lack } \\
\text { of robustness against scale and noise. }\end{array}$ \\
\cline { 2 - 5 } & $\begin{array}{c}\text { Color } \\
\text { correlogram }\end{array}$ & $\begin{array}{l}\text { Similar as above with the adding of the } \\
\text { spatial correlation of every pair of } \\
\text { colors. }\end{array}$ & $\begin{array}{l}\text { Cost computational, not invariant } \\
\text { against the change around of effect, } \\
\text { such as illumination, and scale } \\
\text { effects }\end{array}$ \\
\hline
\end{tabular}




\begin{tabular}{|c|c|c|c|}
\hline \multirow[t]{2}{*}{ Texture } & $\begin{array}{c}\text { Edge } \\
\text { histogram }\end{array}$ & $\begin{array}{l}\text { This descriptor find local feature and is } \\
\text { invariant to for the most part of image } \\
\text { effects, such as transformation }\end{array}$ & $\begin{array}{l}\text { These descriptors do not work fine } \\
\text { with existing non discriminative } \\
\text { feature map (extremely sensitive to } \\
\text { scene and object distortion). }\end{array}$ \\
\hline & $\begin{array}{l}\text { Gabor } \\
\text { filters }\end{array}$ & $\begin{array}{l}\text { used multi-resolution plan for both } \\
\text { classification part and texture feature } \\
\text { as it provide filters based on different } \\
\text { orientation, and size }\end{array}$ & $\begin{array}{l}\text { It construct unnecessary filters; not } \\
\text { every of these filters be able to give } \\
\text { meaningful information cost } \\
\text { computational is high }\end{array}$ \\
\hline \multirow[t]{2}{*}{ Shape } & $\begin{array}{c}\text { Fourier } \\
\text { descriptor }\end{array}$ & $\begin{array}{l}\text { It is invariant to a number of image } \\
\text { effect such as transformation, is strong } \\
\text { against noise, This descriptor give a } \\
\text { distinctive description of shape. }\end{array}$ & $\begin{array}{l}\text { It high computation cost, do not work } \\
\text { good after occlusion occur to shape. }\end{array}$ \\
\hline & SIFT & $\begin{array}{l}\text { It is distinct; does not need } \\
\text { segmentation to get the local feature } \\
\text { and it have invariant } \\
\text { property against transformation effect, } \\
\text { clutter, and occlusion. }\end{array}$ & $\begin{array}{l}\text { The major drawback is } \mathrm{t} \text { high } \\
\text { dimensions feature vector it } \\
\text { construct. }\end{array}$ \\
\hline
\end{tabular}

\section{Images Classification Techniques}

All algorithms that are used in the classification process are determined by the assumption that the data carries one or more features. Generally speaking, classificatory algorithms consist of two successive procedures: training and testing. During the training process, each class is uniquely described by learning the extracted typical features from the sample in question. Then, these extracted features will be separated and confined in the feature space. As for the testing process, in this stage, the separated features will be used in the classification of the new input feature vectors that have been extracted from the testing dataset. In image classification different classification techniques as founded:

A. Decision Tree: Decision tree is a tree-similar graph of decision. all branch represent the decisions to be prepared graphically. It is a non-parametric supervised method. It partition input into regular module. This technique permit the accepted and rejected of class label at all intermediate phase. This way gives the set of rules later than classification that must be understood [18].

B. Support Vector Machine (SVM): In this method, a set of hyperplanes will be constructed in a high dimensional space to be later used in the classification or regression processes. This method is nonparametric and binary classifier; it deals with a large amount of input data efficiently. The accuracy variable is highly affected by hyperplane selection. Moreover, the structure of the algorithm of this method is considered highly complex in comparison with any other methods. That is why; the result of the transparency is low [19].

Artificial Neural Network (ANN): This method is a type of artificial intelligence; it involves emitting several functions of the human mind. This method contains a sequence of layers where every layer of neural network system in return has a group of neurons. The proceeding neurons are connected by weighted connections to the succeeding layers. The accuracy is determined by both the number of the input and the structure of the 
network. Classifying the input using this method is said to be very fast. But, the training process is slow; and the process of choosing the correct architecture is characterized by being tough [20].

Table 2. Show comparison between different classification techniques.

\begin{tabular}{|c|c|c|c|c|}
\hline $\begin{array}{l}\text { Classification } \\
\text { Techniques }\end{array}$ & Advantages & Disadvantages & Complexity & $\begin{array}{l}\text { Performance } \\
\text { and Accuracy }\end{array}$ \\
\hline $\begin{array}{c}\text { Decision } \\
\text { Tree }\end{array}$ & $\begin{array}{l}\text { - This technique does } \\
\text { not require much } \\
\text { effort by the users. } \\
\text { - Easy to explain and } \\
\text { interpret to the user. }\end{array}$ & $\begin{array}{l}\text { - Splits all susceptible } \\
\text { to training data. } \\
\text { - The percentage of } \\
\text { errors resulting in } \\
\text { classification is high. }\end{array}$ & Low & $\begin{array}{l}\text { Depends upon } \\
\text { hierarchical } \\
\text { rule based } \\
\text { technique and } \\
\text { make use of } \\
\text { Nonparametric } \\
\text { approach. }\end{array}$ \\
\hline $\begin{array}{l}\text { Support } \\
\text { Vector } \\
\text { Machine }\end{array}$ & $\begin{array}{l}\text { - Its ability to reach a } \\
\text { unique solution. } \\
\text { - It is an efficient way } \\
\text { in the classification } \\
\text { process. }\end{array}$ & $\begin{array}{l}\text { - The training process } \\
\text { consumes } \\
\text { considerable time. } \\
\text { - The structure of this } \\
\text { algorithm is difficult } \\
\text { to understand. }\end{array}$ & High & $\begin{array}{l}\text { Based on hyper } \\
\text { plane choice } \\
\text { and kernel } \\
\text { parameter. }\end{array}$ \\
\hline $\begin{array}{l}\text { Artificial } \\
\text { Neural } \\
\text { Network }\end{array}$ & $\begin{array}{l}\text { - This technique is one } \\
\text { of the most powerful } \\
\text { ways to train data that } \\
\text { contains noise. } \\
\text { - If the dataset is large, } \\
\text { this technique works } \\
\text { very efficiently. }\end{array}$ & $\begin{array}{l}\text { - Need a lot of time to } \\
\text { train. } \\
\text { - Difficulty choosing } \\
\text { the network structure }\end{array}$ & High & $\begin{array}{ll}\text { Based } & \text { on } \\
\text { network } & \\
\text { architectures } & \\
\text { and } & \\
\text { number } & \text { of } \\
\text { inputs } & \end{array}$ \\
\hline
\end{tabular}

\section{Conclusions}

Image classification plays a key role which is affect by several factors in the field of computer vision. In order to obtain a successful classification system and high accuracy, we must overcome the problems experienced by the classification system that we have focused on through this study, which is the lack of lighting, low resolution and others that are causing the gap between the real image that is classified by humans and the image that is classified by the machine because These factors lose the image a number of characteristics and thus affect the accuracy of the classification, so we try through this study to find solutions to overcome these problems and reduce the gap between the real image and the stage of extracting features from the image .one of these solutions is the selection of a dataset has a key role in overcoming this Problems whenever the dataset is good lead to reduce the gap there are many datasets of them such as Corel datasets and Caltech 101 datasets. and other solutions that we observed through this study The good selection of the method of feature extraction have a important role in influencing the accuracy of the classification and in this study we found many ways to extract features and each method has advantage and disadvantage, The recommendations of this study for the method of color descriptors is the use of histogram color because it 
gives accurate results under certain conditions, As for the texture descriptor, the Cabor filter is recommended because it gives high accuracy and provide multi resolution texture feature, shape descriptor ,SIFT is recommendation are because for use as local descriptors due to the advantages that these descriptors possess . also This survey provide the various classification method with their some limitations. The most commonly used method and reported the best result is the neural network.

\section{Reference}

[1] W.Jianxin,"Efficient SVM learning for image classification", IEEE transaction on image processing, Vol. 21, No. 10, October 2012,

[2] T. Lehmann, M. Guld, T. Deselaers, D. Keysers, H. Schubert, K. Spitzer, H. Ney, and B. Wein "Automatic Categorization of Medical Images for Content-Based Retrieval and Data Mining" Journal of Computerized Medical Imaging and Graphics, Vol.29 ,No.2. pp. 143-155, 2005.

[3] Y. Bengio, A. Courville, and P. Vincent, "Representation learning: a review and new perspectives," IEEE Trans Pattern Anal Mach Intell, vol. 35, pp. 1798-828, Aug 2013.

[4] H. Lee "Unsupervised Feature Learning Via Sparse Hierarchical Representations". Doctor Philosophy, Stanford University,2010.

[5] I. Al Barazanchi, H. R. Abdulshaheed, S. A. Shawkat, and S. R. Binti, "Identification key scheme to enhance network performance in wireless body area network," Period. Eng. Nat. Sci., vol. 7, no. 2, pp. 895-906, 2019.

[7] Z. Abdulelah Al-Sudani, S. Q. Salih, A. Sharafati, and Z. M. Yaseen, "Development of multivariate adaptive regression spline integrated with differential evolution model for streamflow simulation," J. Hydrol., vol. 573, no. 2, pp. 1-12, 2019.

[8] V. V. Thendral Tharmalingam, "An Efficient Convolutional Neural Network Based Classifier to Predict Tamil Writer,” Period. Eng. Nat. Sci., vol. 6, no. 1, pp. 285-295, 2018.

[9] A. S. Abdullah, M. A. Abed, and I. Al Barazanchi, "Improving face recognition by elman neural network using curvelet transform and HSI color space," Period. Eng. Nat. Sci., vol. 7, no. 2, pp. 430-437, 2019.

[10] M. Grubinger,"Analysis and evaluation of visual information systems performance", published of School of Computer Science and Mathematics Faculty of Health, Engineering and Science Victoria University, 2007.

[11] A. Smeulders, M.Worring, S.Santini, A.Gupta, and R. Jain, "Content-based image retrieval at the end of the early years", in IEEE Transactions on Pattern Analysis and Machine Intelligence, Vol.22,No.12: pp. $1349-1380,2000$

[12] P.Stanchev, D. Green and B. Dimitrov. "High level colour similarity retrieval", International Journal of Information Theories and Applications, vol. 10, no. 3, pp. 363-369, 2003.

[13] J. Huang, S. Kuamr, M. Mitra, W. Zhu et al., "Image indexing using colour correlogram", In Proc. Computer Vision and Pattern Recognition, pp. 762-765, 1997. [14] M. Swain and D. Ballard, "Color indexing". International Journal of Computer Vision, vol. 7, no. 1, pp. 11-32, 1991.

[15] D. Tian and B. Shaanxi, "A Review on Image Feature Extraction and Representation", Techniques International Journal of Multimedia and Ubiquitous Engineering, vol. 8, no. 4, pp. 385-396, 2013. 
[16] L. Li and P. Fieguth, "Texture Classification from Random Features," IEEE Transactions on Pattern Analysis and Machine Intelligence, vol. 34, no. 3, pp. 574-586, 2012.

[17] I. Al Barazanchi and H. R. Abdulshaheed, "Adaptive Illumination Normalization Framework based on Decrease Light Effect for Face Recognition,” Jour Adv Res. Dyn. Control Syst., vol. 11, no. 01, pp. 1741-1747, 2019.

[18] D. Zhang and G. Lu, "Review of shape representation and description techniques", Pattern Recognition, vol. 37, no. 1, pp. $1-19,2004$

[19] K. Kumudhaveni , R. Maheswari ," A Survey On Classification Techniques” ,International Journal of New Technologies in Science and Engineering, Vol. 1, No. 3, Sep. 2014.

[20] C. Dhaware K. Wanjale. "Survey On Image Classification Methods In Image Processing", International Journal of Computer Science Trends and Technology - Vol. 4 Issue 3, May - Jun 2016.

[21] J. Ngiam, Z. Chen, D. Chia, P. W. Koh, Q. V. Le, and A. Y. Ng, "Tiled convolutional neural networks," in Advances in Neural Information Processing. 